\title{
Cutaneous Corynebacterium Infection Presenting with Disseminated Skin Nodules and Ulceration
}

\author{
Antonios G.A. Kolios ${ }^{a, b} \quad$ Antonio Cozzio ${ }^{c} \quad$ Annelies S. Zinkernagel ${ }^{d}$ \\ Lars E. French $^{\text {a }}$ Thomas M. Kündig ${ }^{a}$ \\ ${ }^{a}$ Department of Dermatology, University Hospital Zurich, Zurich, Switzerland; \\ ${ }^{b}$ Department of Immunology, University Hospital Zurich, Zurich, Switzerland; \\ ${ }^{\mathrm{C} D e p a r t m e n t}$ of Dermatology and Allergology, Kantonsspital St. Gallen, St. \\ Gallen, Switzerland; ${ }^{d}$ Division of Infectious Diseases and Hospital Epidemiology، \\ University Hospital Zurich, Zurich, Switzerland
}

\section{Keywords}

Cutaneous diphtheria · Corynebacterium diphtheriae · Corynebacterium striatum · Skin ulceration $\cdot$ Skin nodules

\begin{abstract}
In the context of the European migrant crisis, more and more cases of cutaneous diphtheria are seen. A typical presentation includes painful cutaneous ulcerations with grayish-whitish pseudomembranes. Here we present 2 male Eritrean patients suffering from cutaneous nontoxigenic Corynebacterium diphtheriae (patient 1) and Corynebacterium striatum (patient 2) infection.

(c) 2017 The Author(s)

Published by S. Karger AG, Basel
\end{abstract}

\section{Introduction}

Cutaneous diphtheria typically presents as painful cutaneous ulcerations associated with grayish-whitish pseudomembranes and perilesional erythema. Systemic manifestations 
such as pulmonary, gastrointestinal, cardiac and neurological complications have to be ruled out. Since the beginning of the European migrant crisis, more and more cases are seen [1]. The diagnosis may, however, be delayed or even missed since this infection has been rare in developed countries. Here we present 2 patients with a similar clinical presentation of a cutaneous infection with nontoxigenic Corynebacterium diphtheriae (patient 1) and Corynebacterium striatum (patient 2).

\section{Case Report}

We present the cases of 2 male Eritrean patients (patient 1 aged 23 years, patient 2 aged 20 years) with disseminated pruriginous white to grayish hyperkeratotic and in places erosive papules on the trunk, extremities, and penis (Fig. 1a). Patient 2 showed flat cutaneous punched-out-like ulceration with serosanguinous exudate on the left upper leg (Fig. 1b). Both patients were refugees who did not know each other. The skin changes had started independently 2 weeks before consultation while passing through Libya, and subsequently spread slowly over the entire body. Due to the language barrier, a precise and detailed history could not be obtained. Commonly appearing central necrosis was absent in most lesions but typical grayish membranes were present on the erosion and papules. Skin histology showed a superficial to deep dermal eosinophil-rich inflammatory infiltrates with a central ulceration in both patients. PAS/Ziehl-Neelsen and Giemsa stains remained negative. HIV and syphilis serology as well as PCR for Treponema pallidum, Haemophilus ducreyi, and HHV-1/-2 were negative. The gamma-interferon assay was negative in patient 1 and positive in patient 2. Patient 2 also had a left hilar lymphadenopathy on thoracic X-ray but sputum cultures remained negative suggesting latent tuberculosis. Initially, both patients were treated with topical permethrin $5 \%$ and systemic ivermectin due to the suspicion of nodular scabies infestation. However, no typical signs for scabies such as erythematous papules, burrows or dermatoscopic delta signs were found at any of the predilection sites.

Two days after the first consultation, microbiologic cultures of skin swabs revealed C. diphtheriae in patient 1 (Fig. 1a) and C. striatum in patient 2 (Fig. 1b). Both had identical skin lesions. In both cases, the PCR analysis for the toxin was negative and subsequent pharyngeal swab cultures remained negative for $C$. diphtheriae but Streptococcus dysgalactiae grew. Local health authorities were informed. Further investigation did not reveal any systemic organ involvement. Based on the clinical and microbiological presentation the diagnosis of cutaneous Corynebacterium infection was made (cutaneous diphtheria in patient 1) with Staphylococcus aureus superinfection. S. aureus antibiotic susceptibility testing showed resistance to penicillin and susceptibility to erythromycin in both patients. Patients were treated with peroral erythromycin $500 \mathrm{mg}$ twice daily for 14 days. Topical therapy included fusidic acid and medium strength steroids. Within 2-3 weeks, all skin lesions had completely resolved as reported by the treating physician. Unfortunately, no photographs were pro vided.

\section{Discussion}

Cutaneous diphtheria due to both toxigenic and nontoxigenic strains is a reemerging highly contagious skin infection with outbreaks reported in developed countries, affecting persons with poor hygienic status and/or patients with intravenous drug abuse [2,3]. Due to 
the high vaccination rates, it is rather rare in developed Western countries but with increasing migration and the recent wave of refugees in Europe, more and more cases are being seen. It is primarily characterized by nonhealing chronic ulcerations with grayish-whitish pseudomembranes, perilesional erythema and local edema, but rarely eczematous or nodular forms with grayish, yellowish to brownish-gray membranes have also been described. Depending on the route of infection, several forms can be differentiated: first by heteroinoculation, second after autoinoculation (e.g., pharyngeal diphtheria), and third wound diphtheria when a preexisting wound is infected by $C$. diphtheriae. Cutaneous diphtheria lesions are commonly superinfected with $S$. aureus or Streptococcus spp. C. diphtheriae, especially toxinpositive strains, has the potential for systemic infection with pulmonary, gastrointestinal, cardiac and neurological complications [4]. First-line therapy is penicillin or erythromycin but multiresistant Corynebacterium strains have been described [5]. Vaccination with diphtheria and tetanus vaccines protects from the toxin-associated $C$. diphtheriae manifestations but does not have an impact on $C$. diphtheriae colonization. Thus, previous vaccination does not exclude a $C$. diphtheriae infection [6].

C. striatum is commonly found on human skin and mucous membranes and shows emerging evidence as a pathogen in immunocompromised patients or patients with prosthetic devices [7]. Concerns exist as person-to-person transmission has been reported [8]. Pneumonia, endocarditis and septicemia are the most common complications, whereas skin infections (slowly progressive painful skin ulcers), meningitis or central venous catheter infections are rare [9-11]. To our knowledge, this is the first case of a patient showing a nodular variant of $C$. striatum skin infection.

Clinical differential diagnoses include scabies, ecthyma, leishmaniasis, atypical mycobacteriosis, and pyoderma gangrenosum. As both patients were treated topically and systemically for scabies infestation, although diagnostic clinical evidence was missing, a concomitant scabies infestation could not be ruled out completely. If the vaccination status of a patient is uncertain, it is recommended to vaccinate against diphtheria, tetanus, polio and pertussis following the diagnosis with boosters after 4-8 weeks and 6-12 months after the 2nd injection [12]. In case of vaccine-preventable diseases like $C$. diphtheriae, public health interventions are indicated to prevent outbreaks like prevention of infections, restriction of cases, swabs, chemoprophylaxis and vaccination or boosters for all medium to close contacts.

In sum, we report the cases of 2 refugees with similar cutaneous manifestations of Corynebacterium infections presenting with grayish-whitish nodules and cutaneous ulceration without any signs of systemic infection. Both patients were superinfected with $S$. aureus. The skin lesions responded well to systemic erythromycin and resolved completely within 2 weeks. Cutaneous diphtheria should be included in the differential diagnosis of patients with skin ulcerations including the less common grayish papules, especially if occurring in migrants or patients with drug abuse. Cutaneous diphtheria occurring in migrants requires special awareness of both the medical community as well as the attention of public health authorities in order to prevent possible public health hazards due to the high contagiousness [1].

\section{Acknowledgments}

We thank Reinhard Zbinden for critical revision of the manuscript and the technicians of the Institute of Medical Microbiology of the university for their technical assistance. 
Kolios et al.: Cutaneous Corynebacterium Infection Presenting with Disseminated Skin Nodules and Ulceration

\section{Statement of Ethics}

The authors have no ethical conflicts to disclose.

\section{Disclosure Statement}

The authors declare no conflicts of interest.

\section{Author Contributions}

All authors had full access to all of the data in the case. Drs. Kolios and Kündig take responsibility for the integrity of the data and the accuracy of the data analysis. Drafting of the manuscript: Kolios, Kündig. Critical revision of the manuscript for important intellectual content: Cozzio, Zinkernagel, French. Study supervision: Kündig.

\section{References}

1 Meinel DM, Kuehl R, Zbinden R, Boskova V, Garzoni C, Fadini D, et al: Outbreak investigation for toxigenic Corynebacterium diphtheriae wound infections in refugees from Northeast Africa and Syria in Switzerland and Germany by whole genome sequencing. Clin Microbiol Infect 2016;22:1003e1$1003 \mathrm{e} 8$.

2 Lowe CF, Bernard KA, Romney MG: Cutaneous diphtheria in the urban poor population of Vancouver, British Columbia, Canada: a 10-year review. J Clin Microbiol 2011;49:2664-2666.

-3 Gubler J, Huber-Schneider C, Gruner E, Altwegg M: An outbreak of nontoxigenic Corynebacterium diphtheriae infection: single bacterial clone causing invasive infection among Swiss drug users. Clin Infect Dis 1998;27:1295-1298.

-4 Moore LS, Leslie A, Meltzer M, Sandison A, Efstratiou A, Sriskandan S: Corynebacterium ulcerans cutaneous diphtheria. Lancet Infect Dis 2015;15:1100-1107.

5 Livingood CS, Perry DJ, Forrester JS: Cutaneous diphtheria; a report of 140 cases. J Invest Dermatol 1946;7:341-364.

-6 Sing A, Heesemann J: Imported cutaneous diphtheria, Germany, 1997-2003. Emerg Infect Dis 2005;11:343-344.

7 Verma R, Kravitz GR: Corynebacterium striatum empyema and osteomyelitis in a patient with advanced rheumatoid arthritis. BMJ Case Rep 2016;2016:bcr2016214691.

-8 Leonard RB, Nowowiejski DJ, Warren JJ, Finn DJ, Coyle MB: Molecular evidence of person-to-person transmission of a pigmented strain of Corynebacterium striatum in intensive care units. J Clin Microbiol 1994;32:164-169.

-9 Weiss K, Labbe AC, Laverdiere M: Corynebacterium striatum meningitis: case report and review of an increasingly important Corynebacterium species. Clin Infect Dis 1996;23:1246-1248.

10 Watkins DA, Chahine A, Creger RJ, Jacobs MR, Lazarus HM: Corynebacterium striatum: a diphtheroid with pathogenic potential. Clin Infect Dis 1993;17:21-25.

11 Superti SV, Martins Dde S, Caierao J, Soares F, Prochnow T, Cantarelli VV, et al: Corynebacterium striatum infecting a malignant cutaneous lesion: the emergence of an opportunistic pathogen. Rev Inst Med Trop Sao Paulo 2009;51:115-116.

12 Rappold LC, Vogelgsang L, Klein S, Bode K, Enk AH, Haenssle HA: Primary cutaneous diphtheria: management, diagnostic workup, and treatment as exemplified by a rare case report. J Dtsch Dermatol Ges 2016;14:734-736. 


\section{Case Reports in Dermatology}
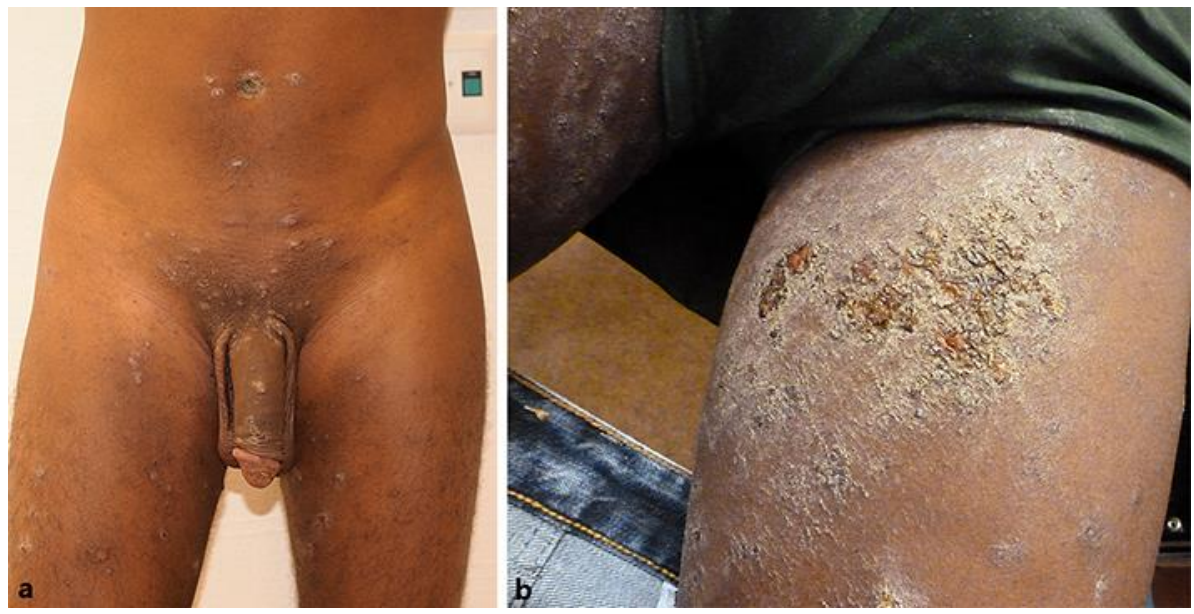

Fig. 1. a Disseminated pruritic white/gray hyperkeratotic papules on the trunk and legs at first presentation (patient 1). b Several flat skin ulcerations with serosanguinous exudate on the left upper leg as well as disseminated pruritic white/gray hyperkeratotic papules (patient 2). Adjustments to brightness, color or contrast has been made to all of the images. 